\title{
Size Control of Gold Nanoparticles During Laser Ablation In Liquids With Different Functional Molecules
}

\author{
Aissam Essaidi ${ }^{* 1}$, M'Barek Chakif ${ }^{{ }^{1} 1}$, Benjamin Schöps ${ }^{{ }^{* 1}}$, Andreas Aumman ${ }^{{ }^{* 1}}$, Shizhou Xiao ${ }^{{ }^{* 1}}$, \\ Cemal Esen ${ }^{* 1}$ and Andreas Ostendorf ${ }^{* 1}$ \\ ${ }^{* 1}$ Ruhr-University Bochum, Applied Laser Technology (LAT), Universitätsstraße 150, 44801 Bochum, \\ Germany. \\ Emails: essaidi@lat.rub.de
}

\begin{abstract}
:
Over the last decade many papers dedicated to the generation of nanoparticles in liquids using laser ablation have been published. They have shown that the nanoparticles size distribution is dependent of the laser fluence and shifts towards smaller sizes when the surfactant concentration is increased. In this paper an alternative approach is presented that permits the control of the size distribution of gold nanoparticles during laser ablation in aqueous solutions containing different surfactant molecules with different shapes and size.

DOI:10.2961/jlmn.2013.02.0003
\end{abstract}

Keywords: Laser ablation, gold nanoparticles, size control, surfactant molecules.

\section{Introduction}

Over the last decade many papers dedicated to the generation of nanoparticles (NPs) in liquids have been published [1-6]. Due to the numerous advantages of this method such as simplicity of the experimental setup, low-cost, environmental friendly etc. this method has become a promising technique for the chemically pure synthesis of NPs in liquid solutions.

The physical and chemical properties of nanoparticles depend on their dimension. Consequently, the control of the particle dimension is an important requirement in terms of applications. In this sense numerous papers were published and the authors have shown that the nanoparticle size distribution depends on the laser fluence, repetition rate, wavelength and shifts towards smaller sizes when the surfactant concentration is increased [5-14]. This effect has been attributed to the gradual binding of the molecules to the metal NPs resulting in a shielding effect and preventing further growth of the particles.

In this paper an alternative approach is presented that permits the control of the size distribution of gold nanoparticles during laser ablation in aqueous solutions containing different molecules. The used molecules consist of two parts: a 'hydrophilic head group' and a 'hydrophobic tail group'. All the molecules exhibit the same head (hydrophilic head: $-\mathrm{SO}_{3}^{-}$) but the body (hydrophobic tail $\mathrm{R}^{\prime}$ or $\mathrm{R}^{\prime \prime}$ ) is different in length and shape. We have studied the influence of the length and the shape of the surfactants on the size distribution of the gold nano- particles prepared by laser ablation in liquids. The laser parameters were the same for all gold nanoparticles colloidal preparation in this work.

\section{Material and experimental procedure}

\section{a. Materials}

All chemicals used in this work were of analytical grade and purchased from Sigma-Aldrich. As surfactants we used:

- $\quad$ Sodium dodecyl sulfate (SDS).

- $\quad$ Eriochrom Black T (EBT).

- $\quad$ Sodium 4-vinylbenzene sulfonate (SVBS).

- $\quad$ Sodium dodecylbenzene sulfonate (SDBS).

As target we used a gold metal plate (99.99\%).

Laser ablation was carried out using a Nd:YAG-Laser (Lumera Laser Hyper Rapid 25) (Fig 1.). The radiation was focused by an objective lens with pulse duration of $15 \mathrm{ps}$ and focal distance of $60 \mathrm{~mm}$ onto the surface of the gold target (fig 1.).

A schematic illustration of the surfactant molecules used in this work is provided in fig 2 .

\section{b. Experimental procedure}

Gold NPs were prepared by laser ablation of a gold metal plate in an aqueous solution. The gold metal plate was placed on the bottom of a glass vessel filled with the specific aqueous solutions with a height of $\sim 10 \mathrm{~mm}$. (fig 2.). 
The surfactants are provided as powder in their initial form and were mixed with distilled water to prepare the aqueous solution. The solution concentrations used in this work are $C_{1}, C_{2}$ and $C_{3}$ for the surfactants SDBS, SDS and SVBS, $\mathrm{C}_{3}$ for EBT $\left(\mathrm{C}_{1}=10^{-1} \mathrm{M}, \mathrm{C}_{2}=10^{-2} \mathrm{M}\right.$ and $\left.\mathrm{C}_{3}=10^{-3} \mathrm{M}\right)$.

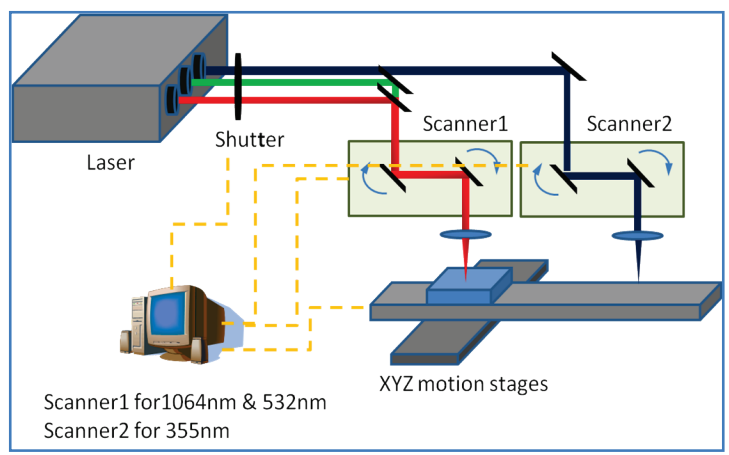

Fig. 1: Schematic setup of the laser system.

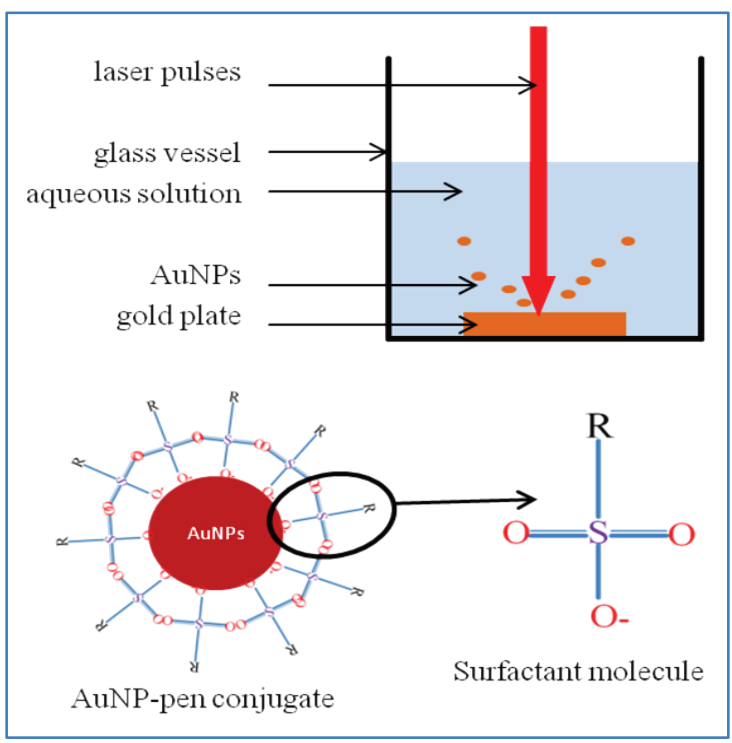

Fig. 2: Preparation of AuNPs by laser ablation and schematic illustration of surfactant.

Table 1 Different surfactants used and their concentrations

\begin{tabular}{|r|c|c|c|}
\hline Concentrations/ & & & \\
Molecules & $\mathrm{C}_{1}$ & $\mathrm{C}_{2}$ & $\mathrm{C}_{3}$ \\
\hline SDBS & $\sqrt{ }$ & $\sqrt{ }$ & $\sqrt{ }$ \\
\hline SVBS & - & $\sqrt{ }$ & $\sqrt{ }$ \\
\hline SDS & $\sqrt{ }$ & $\sqrt{ }$ & $\sqrt{ }$ \\
\hline EBT & - & - & $\sqrt{ }$ \\
\hline
\end{tabular}

The following parameters have not been changed during all the experimental investigation:
- Laser parameters: $(\lambda=1064 \mathrm{~nm}, \quad \mathrm{tp}=15 \mathrm{ps}$, $\mathrm{Ep}=60 \mu \mathrm{J}$, repetition rate $200 \mathrm{kHz}$ ).

- Volume and height of the solution used by formation of gold nanoparticles are $3 \mathrm{ml}$ and $10 \mathrm{~mm}$ respectively.

\section{Results}

As a result of laser ablation of the gold target in different aqueous solutions colloidal gold NPs have been generated. The size distribution of the obtained gold nanoparticles was investigated by Scanning Electron Microscopy (SEM). Figures 4 and 5 show the SEM micrographs and size distribution of gold nanoparticles obtained by laser ablation in different surfactants with different concentrations $\left(\mathrm{C}_{1}, \mathrm{C}_{2}\right.$ and $\left.\mathrm{C}_{3}\right)$ (Tab. 1). With this analyze method, small nanoparticles $(<10 \mathrm{~nm})$ are probably not detectable. To determine the size distribution of small particles, we used the transmission electron microscopy (TEM).

\subsection{AuNPs generation in solutions containing molecules with different size:}

The three surfactants used in this paragraph are represented by molecules which exhibit the same head but a different body-volume (Fig. 3). The surfactants used are EBT, SDBS and SDS and the concentrations were $\mathrm{C}_{1}=10^{-1}, \mathrm{C}_{2}=10^{-2}$ and $\mathrm{C}_{3}=10^{-3} \mathrm{M}$ for SDBS and SDS and $\mathrm{C}_{3}$ for EBT respectively (Tab. 4 ).

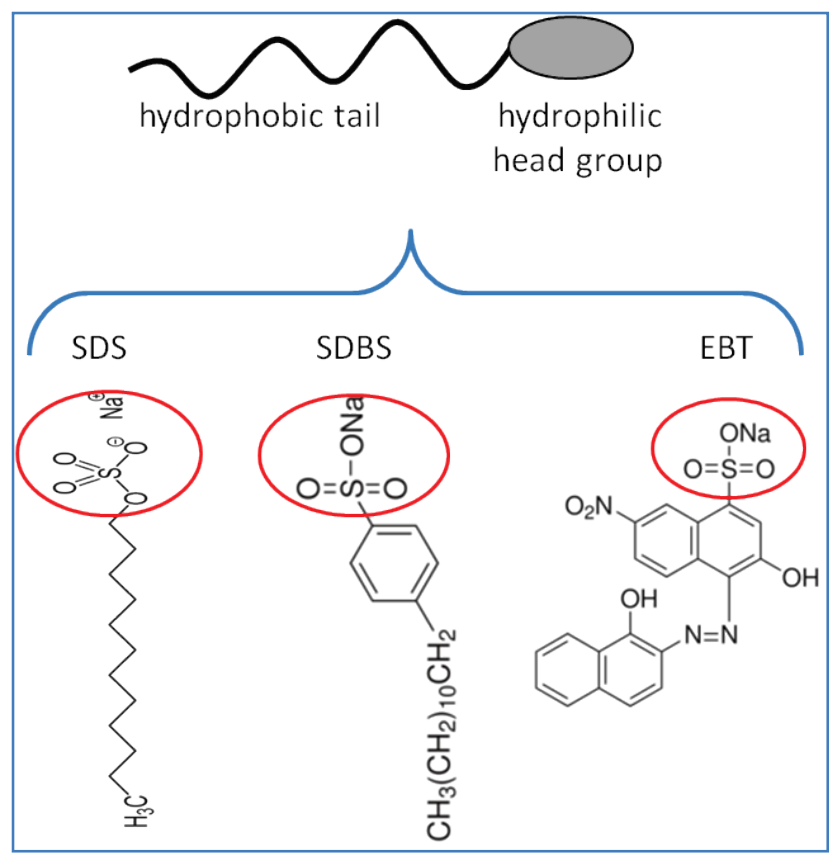

Fig. 3: schematic illustration of surfactant used in this paragraph.

In this paragraph we demonstrate the effect of the molecules' size on the size distribution of the AuNPs prepared by laser ablation. Fig. 4 ( $a, b$ and $c)$ shows the SEM micrograph and size distribution of AuNPs ob- 
tained by laser ablation. To calculate the average diameter of the AuNPs we used at least 2 SEM (or TEM) micrographs for each case. The histograms showed in the figure 4 present some example of the prepared samples. While the table 2 present the calculated average diameter.

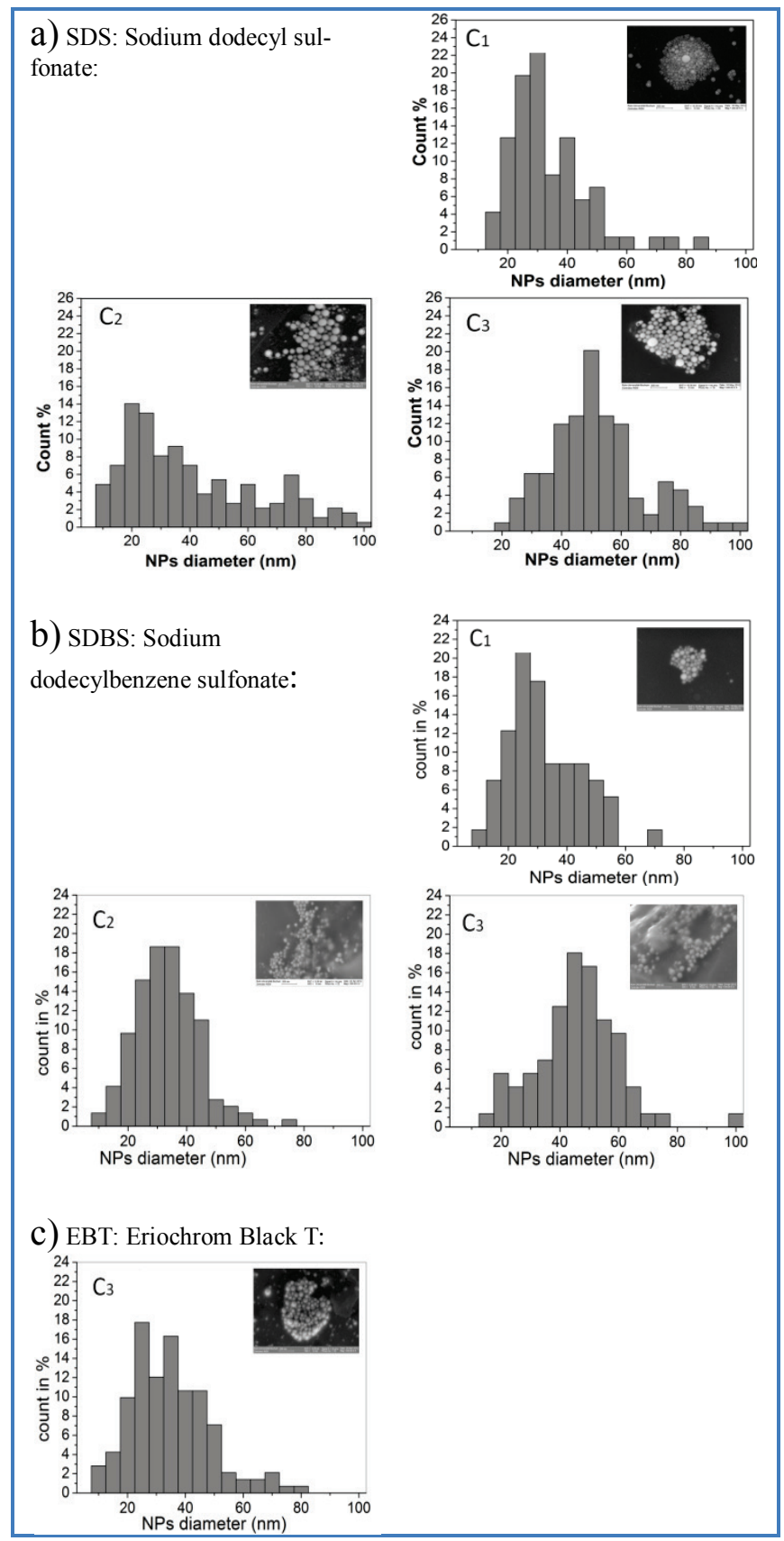

Fig. 4: SEM micrograph and size distribution of prepared AuNPs by laser ablation in concentrations $\left(\mathrm{C}_{1}, \mathrm{C}_{2}\right.$ and $\left.\mathrm{C}_{3}\right)$ of an aqueous solution of a) SDS, b) SDBS and c) EBT.

For SDS, the growth process of nanoparticles depends crucially on the concentration. The average diameter of AuNPs decreases with an increase in the concentration of SDS, the same results have been obtained in references [5-9]. Furthermore, the growth of AuNPs depends on the size of the molecules in the solutions
(Tab. 2). From the schematic illustrations in fig. 3 it can be seen that SDS is basically a smaller molecule compared to SDBS and EBT which is the biggest molecule. The comparison of the resulting average diameter of AuNPs prepared in the solution of SDS, SDBS and EBT with the concentration $\mathrm{C}_{3}$ shows that the average diameter of AuNPs prepared in SDBS is smaller than the AuNPs average diameter obtained in SDS and bigger than that obtained in EBT (Tab. 2).

Table 2 Average diameter of AuNPs obtained in different surfactants with different concentrations.

\begin{tabular}{|c|c|c|}
\hline $\begin{array}{c}\text { Molecules } \\
\text { used in the } \\
\text { solution }\end{array}$ & $\begin{array}{c}\text { Concentrations } \\
(\boldsymbol{M})\end{array}$ & $\begin{array}{c}\text { Average diam- } \\
\text { eter of AuNPs } \\
\text { obtained }(\mathbf{n m})\end{array}$ \\
\hline \multirow{3}{*}{ SDS } & $10^{-1}$ & 34 \\
\cline { 2 - 3 } & $10^{-2}$ & 41 \\
\cline { 2 - 3 } & $10^{-3}$ & 49 \\
\hline \multirow{3}{*}{ SDBS } & $10^{-1}$ & 31 \\
\cline { 2 - 3 } & $10^{-2}$ & 33 \\
\hline \multirow{2}{*}{ EBT } & $10^{-3}$ & 46 \\
\hline
\end{tabular}

\subsection{AuNPs generation in solutions containing molecules with different length:}

The two solutions used in this experimental part contained molecules which have the same head but a different length of the tail (Fig. 5). The surfactants used are SVBS and SDBS and the concentrations were $\mathrm{C}_{1}=10^{-1} \mathrm{M}$, $\mathrm{C}_{2}=10^{-2} \mathrm{M}$ and $\mathrm{C}_{3}=10^{-3} \mathrm{M}$, as before.

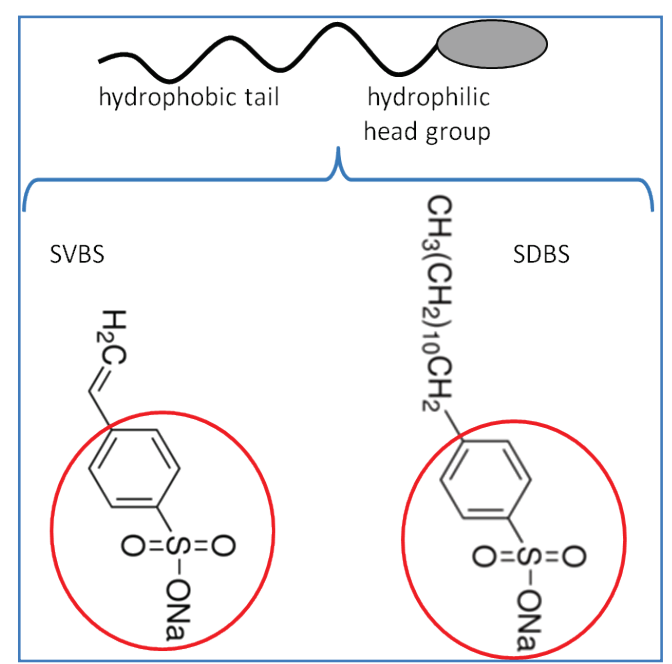

Fig. 5: schematic illustration of surfactant used in this paragraph.

In this paragraph we describe the effect of the length of the surfactant molecules on the size distribution of the gold nanoparticles. Fig. 6 ( $a, b$ and c) shows the SEM micrograph and size distribution of AuNPs obtained by laser ablation. 
a) SVBS: Sodium dodecylbenzene sulfonate
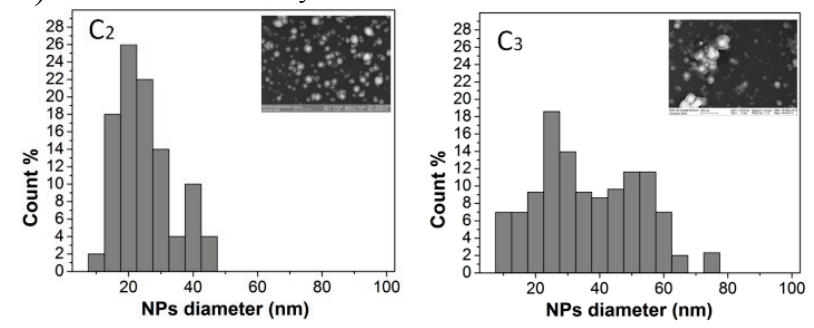

b) SDBS: Sodium dodecylbenzene sulfonate
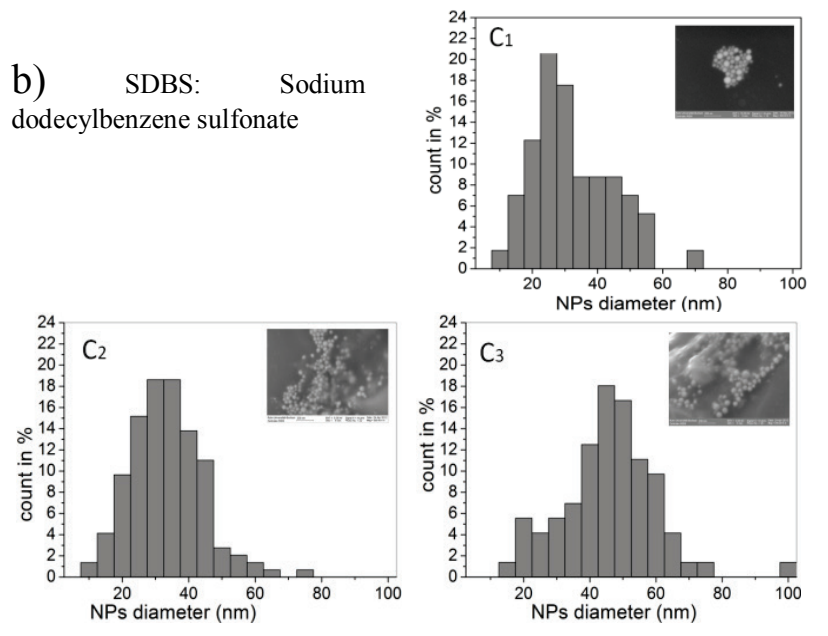

Fig. 6: SEM micrograph and size distribution of prepared AuNPs by laser ablation in concentrations $\left(\mathrm{C}_{1}, \mathrm{C}_{2}\right.$ and $\left.\mathrm{C}_{3}\right)$ of an aqueous solution of a) SDBS, and b) SVBS.

For the two surfactants used, it is obvious that the growth processes of nanoparticles depend crucially on the concentration of the solution in the aqueous solution. In every case the average diameter of gold nanoparticles decreases with an increase in the concentration of the surfactants which confirms the results obtained in references [5-9]. Furthermore, the growth of the AuNPs depends on the length of the molecules in the solutions (Tab. 3):

$>$ In the first case (Fig. 6a) the molecule SVBS is shorter compared to the molecule SDBS used in the second case (Fig. 5).

$>$ The average diameter of the AuNPs prepared in SVBS is smaller than the average diameter of AuNPs obtained in SDBS (Fig. 6a and 6c).

Table 3 Average diameter of AuNPs obtained in different surfactants with different concentrations.

\begin{tabular}{|c|c|c|}
\hline $\begin{array}{c}\text { Molecules } \\
\text { used in the } \\
\text { solution }\end{array}$ & $\begin{array}{c}\text { Concentrations } \\
(\boldsymbol{M})\end{array}$ & $\begin{array}{c}\text { Average diameter } \\
\text { of obtained } \\
\text { AuNPs (nm) }\end{array}$ \\
\hline \multirow{3}{*}{ SDBS } & $10^{-1}$ & 31 \\
\cline { 2 - 3 } & $10^{-2}$ & 33 \\
\cline { 2 - 3 } & $10^{-3}$ & 46 \\
\hline \multirow{2}{*}{ SVBS } & $10^{-2}$ & 30 \\
\cline { 2 - 3 } & $10^{-3}$ & 37 \\
\hline
\end{tabular}

In addition, the size distribution of gold nanoparticles was determined using transmission electron microscopy (TEM) (Fig. 7). To determine the size distribution of small particles, the use of TEM appears most suitable. One observes a significant difference in the size value determined by SEM compared to the ones determined by TEM with a factor of 6 (Fig. 7c and Tab. 4). The particles seem to be larger, due to the nature of the SEM method. As the scattering of the electrons from small particles is weaker compared to larger particles, these are often not detectable by the SEM method [15]. We think that small particles can transmit the electrons for high acceleration voltage $(>10 \mathrm{kV})$ which is the case in our experiment $(\sim 11 \mathrm{kV})$. Similar effect has been observed by Guverich et al. [16]. Also, the presence of organic ligands has an effect on the SEM-measurement, as the molecules adsorbed on the NP surface increase the effective average diameter of the particles when a sample is spattered with a conductive material (layer). In contrast, these molecules do not affect the TEM measurement, as organic materials are transparent for this method. As a consequence small particles are often not visualized and can be inaccurate. S. Petersen et al. have also compared two different measurement methods for analyzing the particle size distribution and founded two different results between TEM and DLS [15]. The average diameter of the particles obtained in SVBS is smaller than the ones obtained in SDBS. This result is shown both with the SEM and with TEM measurement.
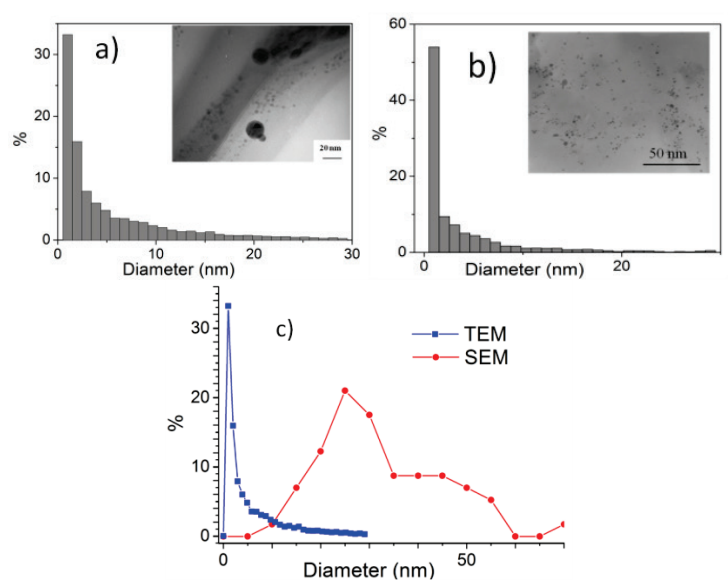

Fig. 7: TEM micrograph and size distribution of AuNPs prepared in the concentrations $\mathrm{C}_{1}$, of an aqueous solution of a) SDBS and b) SVBS. c) Size distribution of gold particles prepared in SDBS with the concentration $\mathrm{C}_{1}$ analyzed by SEM and TEM.

Table 4 Average diameter of AuNPs obtained in the surfactants SDBS analyzed with TEM and SEM.

\begin{tabular}{|c|c|c|}
\hline & Concentrations (M) & SDBS \\
\hline SEM & $\mathrm{C}_{1}$ & 31 \\
\hline TEM & $\mathrm{C}_{1}$ & 5 \\
\hline
\end{tabular}




\section{Discussion}

In the region of high laser intensity on the gold target surface highly excited species and neutrals from the material's surface were ejected. The species were produced in the form of a vastly non-equilibrium, energetic, and dense luminescent plume [6]. The plume vapor has temperatures of the order of $\sim 10,000 \mathrm{~K}$, pressures of a few tenths of GPa and travels with a high speed [6, 17]. By a sufficient amount of the fragmented particles and molecules, a nanoparticle was obtained. A cool-down of the plume results in condensation, nucleation and clustering of the small particles in a very short time scale ( $\sim$ ns) with the formation of NPs up to a certain size $(\sim 1 \mathrm{~nm})$. The electrostatic attachment of this NPs by surfactant molecules via the head group " $-\mathrm{SO}_{3}{ }^{-}$" end with formation of a shell around on the particles. Simultaneously, the nanoparticle gradually grows by attaching more clusters to the particle.

On the other hand, the effect of surfactant on the size and the shape of NPs depends on the type of charge on the NPs' surface and the type of surfactant. For example, an increase in the concentration of SDS was shown to decrease both particle size and size distribution width of surface-charged particles [17]. This effect is due to size quenching of the ablated nanoparticles by coordination of molecules during the nanoparticle nucleation and growth process in the colloidal state. The primary metal nanodroplets were ejected into the liquid medium at a high speed. Due to their compactness, the nanodroplets were shown to react with the ambient medium gradually from the surfaces. X-ray photoelectron spectroscopy of gold nanoparticles observed by Sylvestre et al. revealed the presence of the oxidation states $\mathrm{Au}^{+}$and $\mathrm{Au}^{3+}$ besides the metallic $\mathrm{Au}^{0}$ due to partial oxidation in aqueous media [18]. The degree and rate of such reactions on the particle surfaces were primarily governed by the reactivity between the particles and surfactant molecules and laser parameters, thus leading to products with different morphologies and chemical compositions [17]. Hence, the reactions and final products can be controlled by selecting appropriate surfactants (and/or their concentration). In our experiments we used surfactants which have similar head group $\mathrm{SO}_{3}{ }^{-}$and the same laser parameters to compare the effect of the ligand sizes.

First, the effect of the surfactant's concentration on the size of the obtained gold nanoparticles is discussed (for SDBS molecules for example). After the formation of small particles $(\sim 1 \mathrm{~nm})$ during laser ablation these particles start to be coated by SDBS molecules. In the case of high concentration $(0,1 \mathrm{M}$ or higher), a higher probability result coating of the gold NPs by molecules, i.e. the gold clusters formed afterwards tend to continually attach the nanoparticle. However if the gold nanoparticles are coated by molecules, the growth of the particles is prevented. Consequently they don't have any chance to further grow after the nanoparticle is com- pletely covered by molecules and the growth of the particle is stopped. Therefore, smaller NPs were formed. In the case of low concentration $(0,001 \mathrm{M}$ or lower) in the solution, the probability that gold NPs are coated by molecules is decreased. This could be ascribed to the long time these particles need before they approach SDBS molecules to be coated by them. They have more time to grow. Thus, the resulting AuNPs formed by laser ablation in the low concentration are larger than generated in high concentrations. Peterson et al., demonstrated the influence of ligands also by an ex situ (after laser ablation) laser based conjugation [15].

Secondly, the effect of the shape and length of the surfactant molecules on the AuNPs-growth during laser ablation is discussed:

1. The resulting size distributions of the prepared gold NPs in solutions can be controlled by molecules of different size. If the tail of the surfactant is comparably small (like with SDS for example), more molecules are needed to cover a nanoparticle surface. Similarly, a NP which has the same size can be covered with fewer molecules if these molecules exhibit a large tail like with EBT. This means, more molecules are required to cover the nanoparticle surface if the molecules possess a small hydrophobic tail.

The effective footprint $(\mathrm{K})$ of the surfactants molecules is one way to think about the spatial arrangement of the molecule on the nanoparticle surface. Footprint $(\mathrm{K})$ is defined as the average area each molecule occupies on the nanoparticle surface. The footprint and deflection angles between molecules attached to AuNP can be calculated based on eqs 1-3 from Hill et al. [19], assuming the particles are perfect spheres. The numbers of particles larger and smaller than the average particle size were assumed to be equal, and the surfactants molecules were assumed to be uniformly distributed on the nanoparticle surface [19]. The three eqs are:

$$
\begin{aligned}
& K\left(n m^{2}\right)=\frac{4 \pi r^{2}}{N_{r}} \\
& R=\sqrt{(K / \pi)} \\
& \emptyset(\text { deg })=2 R / r \times 180 / \pi
\end{aligned}
$$

Within the equations, $\mathrm{K}$ represents the ligand's footprint in $\mathrm{nm}^{2}, \mathrm{~N}$ is the average number of ligands per AuNP for a given particle radius $\mathrm{r}, \mathrm{R}$ represents the radius of footprint approximation on the nanoparticle surface, and $\emptyset$ represents the deflection angle in degrees (Fig. 8). For the molecules SDBS the footprint and the deflection angle were be calculated: $\mathrm{K}=0,18 \mathrm{~nm}^{2}, \mathrm{R}=0,24 \mathrm{~nm}$ [20] and $\varnothing$ is $11^{\circ}$. The number of molecules needed to cover $5 \mathrm{~nm}$ particles in the diameter is calculated to be approximately $\sim 436$. 


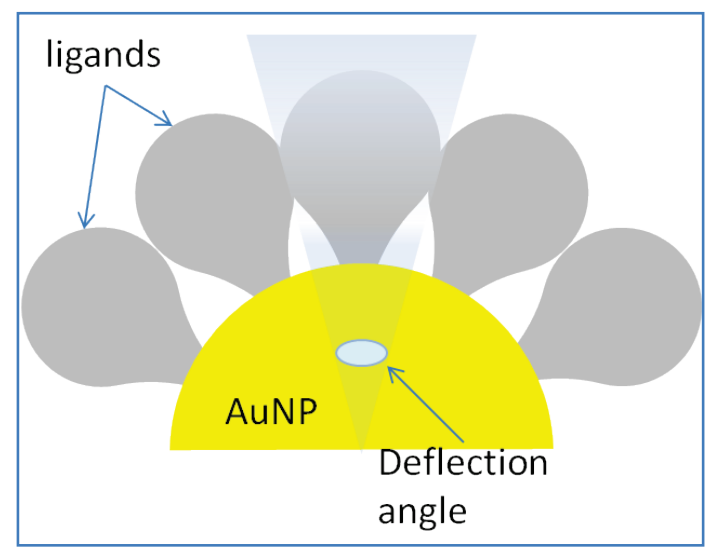

Fig. 8: Illustration of the curvature of the AuNP from surfactants molecules and the deflection angle between the ligands.

2. In addition, we observed an effect of the length of the molecules on the size of gold NPs. In the case, when the surfactant exhibits a long tail the obtained nanoparticles are larger than for shorter molecules. The calculation of the footprint of the ligand SVBS can help to understand this different in the average diameter obtained. The SVBS molecules have the same head group as the SBDS molecules, this means that the molecule SVBS should have the same footprint as with the molecule SDBS. This could be attributed to the low moving velocity of molecules with long tails and therefore a longer time needed to arrive at a nanoparticle surface. In addition, Cederquest et al., have shown that the ligands with long tail can enable the hybridization reaction between the tail regions of the molecules away from the particle surface [21]. This effect can have an impact on the growth of the nanoparticles during laser ablation.

\section{Conclusion}

In conclusion, an alternative approach is presented that permits the control of the size distribution of gold nanoparticles by laser ablation in aqueous solution using surfactants with different size and length. We have demonstrated the possibility to control the size of the nanoparticles during laser ablation using different size of the surfactants. If the molecules are small the nanoparticles obtained are larger than with larger molecules. The size of the nanoparticles depends also on the length of the used surfactant molecules. If the molecule exhibits a short tail (the moving velocity of these molecules in the solution is much higher than for longer molecules) this would result in smaller NPs. Furthermore, the nanoparticles prepared in these solutions are highly stable due to the interaction between the hydrophilic head group "$\mathrm{SO}_{3}{ }^{-“ "}$ of the molecules and the gold nanoparticles surface.

\section{Acknowledgement}

The authors thank: The German Academic Exchange Service (DAAD) for financial support and all members of the Applied Laser Technology department at the RuhrUniversity Bochum.

\section{References}

[1] F. Mafuné, J. Kohno, Y. Takeda, T. Kondow and H. J. Sawabe: Phys. Chem. B, 107, (2003) 4218.

[2] G. W. Yang: Prog. Mater. Sci., 52, (2007) 648.

[3] A. V. Simakin, E. N. Lubnin and G. A. Shafeev: Quantum Electron, 30, (2000) 263.

[4] A. V. Kabashin, M. J. Meunier: Phys.: Conf. Ser., 59, (2007) 354.

[5] F. Mafuné, J. Kohno, Y. Takeda, and T. Kondow: J. Phys. Chem. B, 104, (2000) 9111-9117.

[6] N. G. Semaltianos: Critical Reviews in Solid State and Materials Sciences, 35, (2010) 105-124.

[7] A. V. Simakin, V. V. Voronov and G. A. Shafeev: Physics of Wave Phenomena, 15, (2007) 218-240.

[8] A. Schwenke, P. Wagener, S. Nolte and S. Barcikowski: Applied Physics A, 104, (2011) 77-82.

[9] J. Perrière, C. Boulmer-Leborgne, R. Benzerga and S. Tricot: Journal of Physics D: Applied Physics, 40, (2007) 7069-7076.

[10]F. Mafuné, J. Kohno, Y. Takeda, and T. Kondow: J. Phys. Chem. B, 105, (2001) 5114-5120.

[11] A. V. Kabashin, M. Meunier, C. Kingston, and J. H. T. Luong: J. Phys. Chem. B, 107, (2003) 4527-4531.

[12]E. Akman, B. Genc Oztoprak, M. Gunes, E. Kacar and A. Demir: Photonics and NanostructuresFundamentals and Applications, 9, (2011), 276-286.

[13]A. M. manjón and S. Barcikowski: Appl. Surf. Sci., 9, (2011), 4285-4290.

[14]T. Tsuji, K. Iryo, Y. Nishimura and M. Tsuji: J. Photochemistry and Photobiol. A: Chem., 145, (2001), 201-207.

[15]Petersen et al., Adv. Funct. Mater. (2009), 19,1

[16] E. L. Gurevich and R. Hergenröder, Nanotechnology, (2009), 20.

[17]Zeng et al. Adv. Funct. Mater. (2012), 22, 1333.

[18] Sylvestre, J. P.; Poulin, S.; Kabashin, A. V.; Sacher, E.; Meunier, M.; Luong, J. H. T. J. Phys. Chem. B (2004), 108, 16864

[19] Hill et al., ACS NANO (2009), 2, 418

[20] J. E. House, Inorganic chemistry - Textbooks I, (2008), 546

[21] Cederquest et al., ACS NANO (2009), 2, 256.

(Received: July 3, 2012, Accepted: February 21, 2013) 